\title{
Spin effects in neutrino gravitational scattering
}

\author{
Maxim Dvornikov@* \\ Pushkov Institute of Terrestrial Magnetism, Ionosphere and Radiowave Propagation (IZMIRAN), \\ 108840 Moscow, Troitsk, Russia
}

(Received 20 November 2019; accepted 8 March 2020; published 26 March 2020)

\begin{abstract}
We study spin oscillations of neutrinos gravitationally scattered off a nonrotating black hole (BH). We derive the transition and survival probabilities of spin oscillations in quadratures when neutrinos interact with BHs only. The dependence of the probabilities on the impact parameter is analyzed. Then, we obtain the effective Schrödinger equation for spin oscillations in neutrino scattering off $\mathrm{BHs}$ surrounded by background matter. This equation is solved numerically in the case of a supermassive BH with a realistic accretion disk. We find that the observed neutrino fluxes can be reduced almost $20 \%$ because of spin oscillations when neutrinos experience gravitational scattering. The neutrino interaction with an accretion disk results in the additional asymmetry in the intensities of outgoing fluxes depending on the neutrino trajectory.
\end{abstract}

DOI: 10.1103/PhysRevD.101.056018

\section{INTRODUCTION}

The experimental observation of neutrino oscillations, reported, e.g., in Ref. [1], confirmed that neutrinos are massive particles having nonzero mixing between different flavors. Among various types of oscillations, we distinguish neutrino spin oscillations [2], which are the transitions between different helicity states within one neutrino type. If a left polarized neutrino changes its polarization, it cannot be observed since right neutrinos are sterile in the standard model. It results in the effective reduction of the initial neutrino flux.

It is known that external backgrounds, e.g., the neutrino interaction with matter [3], can modify the process of neutrino oscillations. The gravitational interaction was found in Ref. [4] to influence flavor oscillations of neutrinos. Neutrino spin oscillations in various external fields in curved spacetime were studied in Refs. [5-7]. We considered both static metrics and time-dependent backgrounds, like a gravitational wave. Note that the evolution of the fermion spin in curved spacetime was analyzed in Refs. [8,9] using both quasiclassical and quantum approaches.

The gravity-induced neutrino spin oscillations, studied in Refs. [5,6,10-12], were analyzed for neutrinos orbiting a massive object, e.g., a black hole (BH). However, in this situation, even if neutrino spin oscillations can be quite

\footnotetext{
maxdvo@izmiran.ru
}

Published by the American Physical Society under the terms of the Creative Commons Attribution 4.0 International license. Further distribution of this work must maintain attribution to the author(s) and the published article's title, journal citation, and DOI. Funded by SCOAP ${ }^{3}$. intense, it is rather difficult to understand what kind of observational effects one can expect, since a particle is gravitationally captured by a $\mathrm{BH}$ or a neutrino falls to the $\mathrm{BH}$ surface. That is why it is interesting to study spin effects or neutrino spin oscillations, e.g., in the neutrino gravitational scattering, when one can control the helicities of both incoming and outgoing particles.

This research is inspired by the recent observation of the shadow of a supermassive BH (SMBH) [13], which provides the unique test of the general relativity in the strong field limit. A bright ring around a $\mathrm{BH}$ shadow is formed by photons, which are emitted by an accretion disk and then experience strong lensing in the gravitational field of the BH [14]. However, in addition to photons, a significant flux of neutrinos was found in Ref. [15] to be emitted by an accretion disk. These particles are subject to neutrino oscillations. In this work, we shall examine how a strong gravitational field of a $\mathrm{BH}$ and the neutrino interaction with an accretion disk can modify the helicity of scattered particles.

The neutrino gravitational scattering was studied recently [16], mainly in connection to the determination of the BH shadow produced by these particles [17]. In our work, we shall focus on the analysis of spin oscillations in the neutrino gravitational scattering, which effectively reduce the flux of neutrinos measured in a detector.

Photons, which form the ring around the $\mathrm{BH}$ shadow, interact with both its gravitational field and plasma that surrounds the $\mathrm{BH}$. The interaction with plasma can modify the size and the form of the BH shadow (see Ref. [18] for a review). In the present work, we shall study how the neutrino interaction with background matter, e.g., with an accretion disk, can influence the observed flux of gravitationally scattered neutrinos. 
In this work, we continue our studies of neutrino spin oscillations from Refs. [5-7]. We start in Sec. II with the analysis of the neutrino spin evolution when a particle gravitationally scatters off a nonrotating $\mathrm{BH}$. We find the expressions in quadratures for the transition and survival probabilities for ultrarelativistic neutrinos and analyze them for different impact parameters. Then, in Sec. III, we formulate the effective Schrödinger equation for neutrino spin oscillations in the scattering off a $\mathrm{BH}$ surrounded by background matter. We study astrophysical applications in Sec. IV. In particular, we consider the effect of spin oscillations on the measured neutrino fluxes when particles scatter off a SMBH with a realistic accretion disk. Finally, in Sec. V, we discuss our results. We remind the reader how a scalar particle moves in the Schwarzschild metric in Appendix.

\section{NEUTRINO SPIN EVOLUTION IN SCATTERING OFF A BH}

In this section, we study how the spin of a neutrino evolves when a particle scatters off a Schwarzschild BH. We solve the spin evolution equation in quadratures and analyze the solution for ultrarelativistic neutrinos. The transition and survival probabilities for neutrino spin oscillations are derived.

We study the neutrino motion in the field of a nonrotating $\mathrm{BH}$. Using the spherical coordinates $(r, \theta, \phi)$, the interval in this case has the form ([19], p. 284)

$$
\mathrm{d} \tau^{2}=A^{2} \mathrm{~d} t^{2}-A^{-2} \mathrm{~d} r^{2}-r^{2}\left(\mathrm{~d} \theta^{2}+\sin ^{2} \theta \mathrm{d} \phi^{2}\right),
$$

where $A=\sqrt{1-r_{g} / r}, r_{g}=2 M$ is the gravitational radius, and $M$ is the BH mass. Since the Schwarzschild metric in Eq. (2.1) is spherically symmetric, we can take that a neutrino moves in the equatorial plane with $\theta=\pi / 2$, i.e., $\mathrm{d} \theta=0$.

In Refs. [5,6], we found that the neutrino invariant $\operatorname{spin} \zeta$, defined in a locally Minkowskian frame, evolves as

$$
\frac{\mathrm{d} \zeta}{\mathrm{d} t}=\frac{2}{\gamma}\left(\boldsymbol{\zeta} \times \boldsymbol{\Omega}_{g}\right)
$$

where $\gamma=\mathrm{d} t / \mathrm{d} \tau$. If a neutrino interacts with a Schwarzschild BH, the vector $\boldsymbol{\Omega}_{g}$ in Eq. (2.2) has only one nonzero component [5],

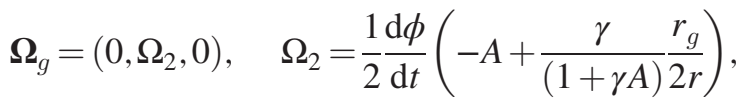

where $\mathrm{d} \phi / \mathrm{d} t=L A^{2} / E r^{2}$ is the angular velocity, which can be obtained using Eq. (A1), $L$ is the conserved angular momentum of a neutrino, and $E$ is the neutrino energy. The parameter $\gamma$ in Eqs. (2.2) and (2.3) has the form $\gamma=E / m A^{2}$, where $m$ is the neutrino mass.
We are interested in neutrino spin oscillations, i.e., in the change of the neutrino helicity, $h=(\zeta \mathbf{u}) /|\mathbf{u}|$, where $\mathbf{u}$ is the spatial part of the neutrino four velocity in the locally Minkowskian frame. Therefore, besides the study of the neutrino spin in Eq. (2.2), we should account for the evolution of $\mathbf{u}$.

The expression for $\mathbf{u}$ in the Schwarzschild metric has the form [5]

$$
\begin{aligned}
\mathbf{u} & =\left(\frac{\mathrm{d} r}{\mathrm{~d} \tau} A^{-1}, 0, r \frac{\mathrm{d} \phi}{\mathrm{d} \tau}\right) \\
& =\left( \pm \frac{1}{m}\left[E^{2}-m^{2} A^{2}\left(1+\frac{L^{2}}{m^{2} r^{2}}\right)\right]^{1 / 2}, 0, \frac{L}{m r}\right),
\end{aligned}
$$

where the signs \pm stay for outgoing and incoming neutrinos, respectively [see Eq. (A1)]. At $r \rightarrow \infty, \mathbf{u} \rightarrow \mathbf{u}_{ \pm \infty}=$ $\left( \pm\left[E^{2}-m^{2}\right]^{1 / 2} / m, 0,0\right)$; i.e., the asymptotic neutrino motion happens along the first axis in the locally Minkowskian frame. In this frame, an incoming neutrino propagates opposite of the first axis. An outgoing particle moves along this axis.

Since only $\Omega_{2} \neq 0$, the nonzero neutrino spin components are $\zeta_{1,3} \neq 0$ and $\zeta_{2}=0$. It is convenient to represent

$$
\zeta_{1}=\cos \alpha, \quad \zeta_{3}=\sin \alpha,
$$

where $\alpha$ is the rotation angle of the spin with respect to its initial direction.

Now we have to specify the initial condition for Eq. (2.2). We suppose that, initially, at $r \rightarrow \infty$ and $\phi \rightarrow 0$, an incoming neutrino is left polarized; i.e., the helicity is negative, $h_{-\infty}=\left(\zeta_{-\infty} \mathbf{u}_{-\infty}\right) /\left|\mathbf{u}_{-\infty}\right|=-1$. Accounting for the expression for $\mathbf{u}_{-\infty}$ above, we get that $\zeta_{-\infty 1}=1$ and $\zeta_{-\infty 3}=0$, or $\alpha_{-\infty}=0$ in Eq. (2.5).

The helicity of an outgoing neutrino has the form $h_{+\infty}=$ $\left(\boldsymbol{\zeta}_{+\infty} \mathbf{u}_{+\infty}\right) /\left|\mathbf{u}_{+\infty}\right|$, where $\boldsymbol{\zeta}_{+\infty}=\left(\cos \alpha_{+\infty}, 0, \sin \alpha_{+\infty}\right)$ and $\mathbf{u}_{+\infty}$ is given above. Using Eq. (2.5), we get that $h_{+\infty}=\cos \alpha_{+\infty}$. The transition $P_{\mathrm{LR}}$ and survival $P_{\mathrm{LL}}$ probabilities for neutrino spin oscillations are

$$
P=\frac{1}{2}\left(1 \pm h_{+\infty}\right),
$$

where the upper sign stays for $P_{\mathrm{LR}}$ and the lower one for $P_{\mathrm{LL}}$.

The angle $\alpha$ corresponds to the spin projection on the $x$ axis in the neutrino rest frame. This projection is in $m / E$ times shorter for a nonmoving observer, which measures the neutrino polarization, because of the Lorentz contraction. It means that the observed angle should be rescaled by the factor $E / m: \alpha \rightarrow \alpha E / m$. It is equivalent to the replacement of $\Omega_{2}: \Omega_{2} \rightarrow \Omega_{2} E / m$.

Now we should find $\alpha_{+\infty}$. Using Eqs. (2.2), (2.3), (2.5), and (A1), we get that the angle $\alpha$ obeys the equation 


$$
\begin{aligned}
\frac{\mathrm{d} \alpha}{\mathrm{d} r} & =F, \\
F(r) & = \pm \frac{A L}{m r^{2}} \frac{\frac{E}{m}\left(\frac{3 r_{g}}{2 r}-1\right)-A^{3}}{\frac{E}{m}+A}\left[\frac{E^{2}}{m^{2}}-A^{2}\left(1+\frac{L^{2}}{m^{2} r^{2}}\right)\right]^{-1 / 2},
\end{aligned}
$$

where the signs \pm stay for outgoing and incoming neutrinos. Then, we should account for the initial condition $\alpha_{-\infty}=0$ and the fact that $\alpha_{+\infty}$ is twice the angle corresponding to the minimal distance between a neutrino and $\mathrm{BH}$. We express the final result for ultrarelativistic neutrinos, with $E \gg m$, as

$$
\alpha_{+\infty}=y \int_{x_{m}}^{\infty} \frac{\mathrm{d} x}{x^{2}} \frac{(3-2 x) \sqrt{x-1}}{\sqrt{x^{3}-y^{2}(x-1)}},
$$

where $y=b / r_{g}, b=L / E$ is the impact parameter, and $x_{m}$ is the maximal root of the equation

$$
x^{3}-y^{2}(x-1)=0 .
$$

Note that $y>y_{0}=3 \sqrt{3} / 2$ for a neutrino not to fall to the BH (see Appendix).

The expression for the roots $x_{1,2,3}$ of Eq. (2.9) for the arbitrary $y$ has the form

$$
\begin{gathered}
x_{k}=\frac{2 y}{\sqrt{3}} \cos \left[\frac{1}{3} \arccos \left(-\frac{3 \sqrt{3}}{2 y}\right)-\frac{2 \pi}{3}(k-1)\right], \\
k=1,2,3,
\end{gathered}
$$

where $x_{1} \equiv x_{m}$ is the maximal root. First, let us analyze Eq. (2.8) in the case $y \gg y_{0}$. Using Eq. (2.10) and keeping only the leading terms, we get that the roots have the form $x_{1}=y-\frac{1}{2}-\frac{3}{8 y}+\mathcal{O}\left(y^{-3}\right), \quad x_{2}=1+\mathcal{O}\left(y^{-4}\right)$, and $x_{3}=-y-\frac{1}{2}+\frac{3}{8 y}+\mathcal{O}\left(y^{-3}\right)$. In this case, we get that

$\alpha_{+\infty}=8 y \int_{a}^{\infty} \frac{\mathrm{d} x}{(2 x-1)^{2}} \frac{(1-x)}{\sqrt{x^{2}-a^{2}}} \approx-\pi-\frac{\pi}{4 y^{2}}$,

where $a=y-\frac{3}{8 y}$. The transition and survival probabilities in Eq. (2.6) take the form

$$
\begin{aligned}
& P_{\mathrm{LR}}=\frac{1}{2}\left[1-\cos \frac{\pi}{4 y^{2}}\right] \approx \frac{\pi^{2}}{64 y^{4}}, \\
& P_{\mathrm{LL}}=\frac{1}{2}\left[1+\cos \frac{\pi}{4 y^{2}}\right] \approx 1-\frac{\pi^{2}}{64 y^{4}} .
\end{aligned}
$$

One can see that $P_{\mathrm{LR}} \rightarrow 0$ (and $P_{\mathrm{LL}} \rightarrow 1$ ) if $y \gg y_{0}$. It is expected since, at $y \gg y_{0}$, a neutrino propagates far away from the BH. The gravitational interaction, which causes the spin flip, is weak; thus, the neutrino polarization is practically unchanged.
Now we discuss the situation when $y \rightarrow y_{0}$. Then, Eq. (2.9) has the following roots: $x_{1}=x_{2}=3 / 2$ and $x_{3}=-3$. The spin rotation angle takes the value

$$
\alpha_{+\infty}=-3 \sqrt{3} \int_{3 / 2}^{\infty} \frac{\mathrm{d} x}{x^{2}} \frac{\sqrt{x-1}}{\sqrt{x+3}}=-\frac{2 \pi}{3},
$$

which is finite even if a neutrino asymptotically approaches $\mathrm{BH}$. The corresponding probabilities are $P_{\mathrm{LR}}=0.25$ and $P_{\mathrm{LL}}=0.75$ for such neutrinos. We shall present the transition and survival probabilities for arbitrary $y$ in Sec. IV, when we study some possible astrophysical applications.

\section{NEUTRINO GRAVITATIONAL SCATTERING ACCOUNTING FOR THE MATTER INTERACTION}

In this section, we formulate the neutrino spin evolution equation in background matter under the influence of a gravitational field when a neutrino scatters off a $\mathrm{BH}$. Then, we derive the effective Schrödinger equation for scattered neutrinos.

Using the forward scattering approximation, one gets that the neutrino interaction with background matter is described by the following effective Lagrangian in Minkowski spacetime [20]:

$$
\mathcal{L}_{m}=-\sqrt{2} G_{\mathrm{F}} \bar{\nu} \gamma^{\mu}\left(1-\gamma^{5}\right) \nu G_{\mu},
$$

where $\nu$ is the neutrino bispinor, $\gamma^{\mu}$ and $\gamma^{5}$ are the Dirac matrices, and $G_{\mathrm{F}}=1.17 \times 10^{-5} \mathrm{GeV}^{-2}$ is the Fermi constant. The four-vector $G^{\mu}$ is the linear combination of the hydrodynamic currents and polarizations of background fermions. It depends on the chemical composition of matter and the type of the neutrino. The explicit form of $G^{\mu}$ can be found in Ref. [21].

Based on Eq. (3.1), the influence of the neutrino interaction with background matter on its spin evolution in curved spacetime was studied in Refs. [6,7]. It results in the appearance of the additional components of the vector $\boldsymbol{\Omega}_{g}$ in Eq. (2.3): $\boldsymbol{\Omega}_{g} \rightarrow \boldsymbol{\Omega}=\boldsymbol{\Omega}_{g}+\boldsymbol{\Omega}_{m}$. If we study the neutrino interaction with nonmoving and unpolarized background fermions in curved spacetime, the vector $\boldsymbol{\Omega}_{m}$ has the form

$$
\boldsymbol{\Omega}_{m}=\frac{G_{\mathrm{F}}}{\sqrt{2}} \frac{g^{0}}{\gamma} \mathbf{u}=\frac{G_{F}}{\sqrt{2}} n_{\mathrm{eff}} \frac{1}{\gamma}\left(\frac{\mathrm{d} r}{\mathrm{~d} \tau}, 0, A r \frac{\mathrm{d} \phi}{\mathrm{d} \tau}\right),
$$

where $g^{0}=e_{\mu}^{0} G^{\mu}=A G^{0}, e_{\mu}^{0}=(A, 0,0,0)$ is the vierbein vector in the Schwarzschild metric (see Ref. [5]), and $G^{0}=$ $n_{\text {eff }}$ is the invariant effective density of background matter. We use Eq. (2.4) to derive Eq. (3.2).

If we study spin oscillations of electron neutrinos in the electrically neutral hydrogen plasma, then $n_{\text {eff }}=n_{e}$, where $n_{e}$ is the electron number density. The expressions for $n_{\text {eff }}$ 
for other neutrino oscillations' channels and various types of background fermions can be found in Ref. [21].

Instead of dealing with Eq. (2.2) for the spin precession, it is convenient to study the neutrino polarization density matrix $\rho=\frac{1}{2}[1+(\sigma \zeta)]$, which obeys the equation $\mathrm{i} \dot{\rho}=[H, \rho]$, where $H=-(\boldsymbol{\sigma} \boldsymbol{\Omega})$ and $\boldsymbol{\Omega}$ includes both the gravity and matter contributions in Eqs. (2.3) and (3.2). Here $\sigma$ are the Pauli matrices.

Since the Liouville-von Neumann equation for the density matrix is rather complicated for the analysis, we can use the Schrödinger equation $\mathrm{i} \psi \mathrm{r}=H \psi$. As we mentioned in Sec. II, neutrinos move along the first axis in the locally Minkowskian frame at $r \rightarrow \infty$. Hence, it is convenient to use this axis for the spin quantization. It means that we should transform the Hamiltonian $\mathrm{H} \rightarrow \mathrm{U}_{2} \mathrm{HU}_{2}^{\dagger}$, where $U_{2}=\exp \left(\mathrm{i} \pi \sigma_{2} / 4\right)$. This procedure brings meaning to the effective wave function $\psi$.

As in Eq. (2.7), it is convenient to rewrite the Schrödinger equation using the radial coordinate $r$,

$$
\mathrm{i} \frac{\mathrm{d} \psi}{\mathrm{d} r}=H_{r} \psi, \quad H_{r}=-U_{2}\left(\boldsymbol{\sigma} \boldsymbol{\Omega}_{r}\right) U_{2}^{\dagger},
$$

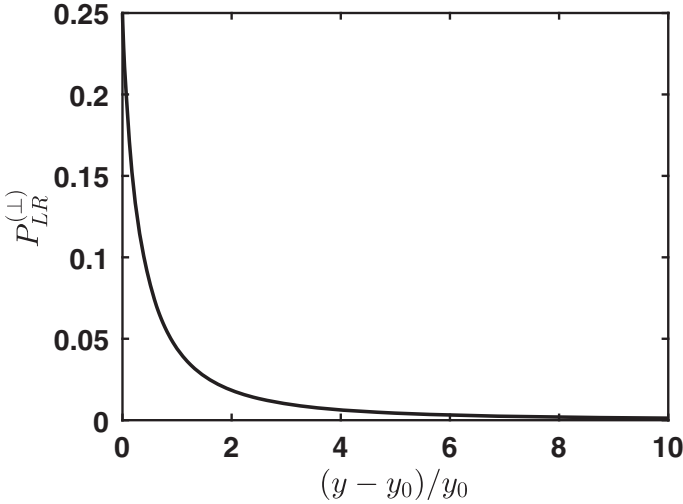

(a) where

$$
\boldsymbol{\Omega}_{r}=\frac{\mathrm{d} t}{\mathrm{~d} r} \boldsymbol{\Omega}=\left(\frac{G_{\mathrm{F}}}{\sqrt{2}} n_{\mathrm{eff}}, \frac{F}{2}, A r \frac{\mathrm{d} \phi}{\mathrm{d} r} \frac{G_{\mathrm{F}}}{\sqrt{2}} n_{\mathrm{eff}}\right)
$$

Here $F$ is given in Eq. (2.7).

Equation (3.3) should be supplied with the initial condition $\psi_{-\infty}^{\mathrm{T}}=(1,0)$, which means that all incoming neutrinos are left polarized. Since the neutrino velocity changes the direction at $t \rightarrow+\infty$, the transition probability reads $P_{\mathrm{LR}}=\left|\psi_{+\infty}^{(1)}\right|^{2}$, and correspondingly, the survival probability is $P_{\mathrm{LL}}=\left|\psi_{+\infty}^{(2)}\right|^{2}$, where $\psi_{+\infty}^{\mathrm{T}}=\left(\psi_{+\infty}^{(1)}, \psi_{+\infty}^{(2)}\right)$ is the asymptotic solution of Eq. (3.3).

The solution of Eqs. (3.3) and (3.4) can be found only numerically because of the nontrivial dependence of $\boldsymbol{\Omega}_{r}$ on $r$. Moreover, in Sec. IV, we discuss the situation when $n_{\text {eff }}=n_{\text {eff }}(r)$, which makes the analysis more complicated.

We also mention that we cannot integrate Eqs. (3.3) and (3.4) to the turn point $r_{m}$ and then automatically reconstruct $\psi_{+\infty}$, as we did in Sec. II to find $\alpha_{+\infty}$. In the presence of the background matter, the dynamics of the neutrino

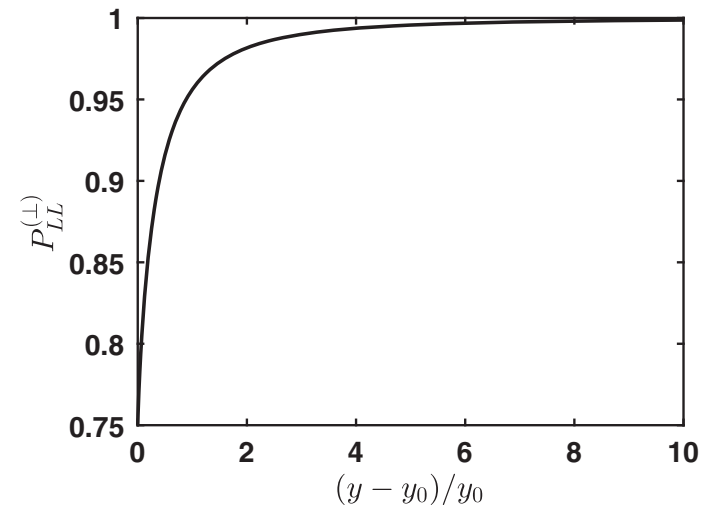

(b)

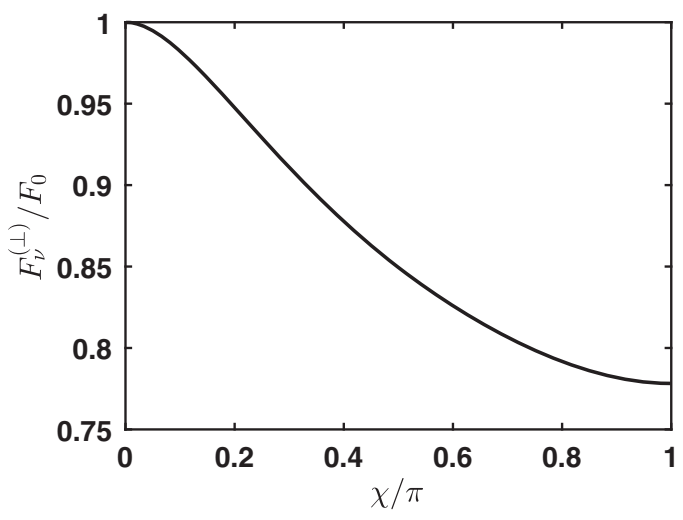

(c)

FIG. 1. (a) The transition probability of spin oscillations $P_{\mathrm{LR}}^{(\perp)}$, when a neutrino moves perpendicular to an accretion disk versus the dimensionless impact parameter $y$. (b) The survival probability of spin oscillations $P_{\mathrm{LL}}^{(\perp)}$ when a neutrino moves perpendicular to an accretion disk, as a function the dimensionless impact parameter $y$. (c) The ratio of the measured fluxes of neutrinos and scalar particles, when they move perpendicular to an accretion disk versus the scattering angle $\chi$ normalized by $\pi$. 
polarization is non-Abelian. Moreover, the term $\frac{\mathrm{d} \phi}{\mathrm{d} r}$ in $\boldsymbol{\Omega}_{r}$ changes the sign at $r_{m}$ [see Eq. (A1)]. Thus, to obtain $\psi_{+\infty}$, one should integrate Eqs. (3.3) and (3.4): first, in the interval $+\infty>r>r_{m}$ and then for $r_{m}<r<+\infty$, with the solutions being stitched at $r_{m}$. This fact significantly reduces the accuracy of the numerical simulation compared to Sec. II.

\section{ASTROPHYSICAL APPLICATIONS}

In this section, we present the numerical solutions of Eqs. (3.3) and (3.4) for the neutrino scattering off a SMBH surrounded by an accretion disk. We discuss different orientations of neutrino trajectories with respect to the disk plane. The measurable neutrino fluxes are obtained.

First, we notice that standard model neutrinos are produced as left polarized particles. If they gravitationally interact with the $\mathrm{BH}$, some incoming left neutrinos become right polarized after scattering. A neutrino detector can observe only left neutrinos. Hence, the observed flux of neutrinos is $F_{\nu}=P_{\mathrm{LL}} F_{0}$, where $F_{0}$ is the flux of scalar particles. The value of $F_{0}$ is proportional to the differential cross section $F_{0} \sim \mathrm{d} \sigma / \mathrm{d} \Omega$, which is studied in Appendix.

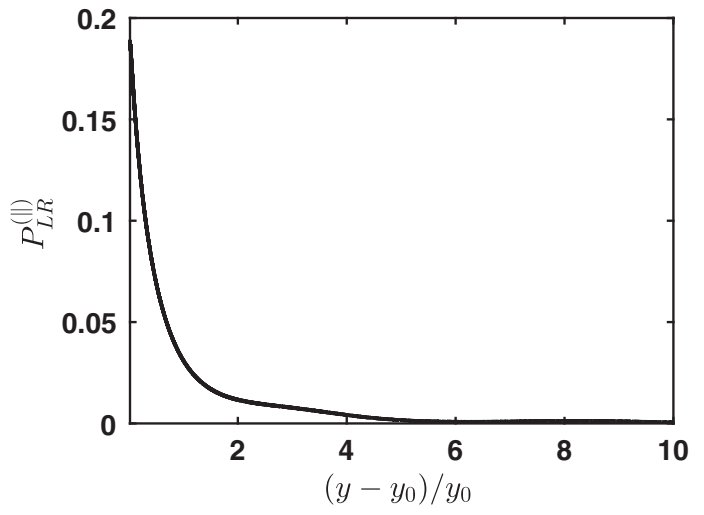

(a)

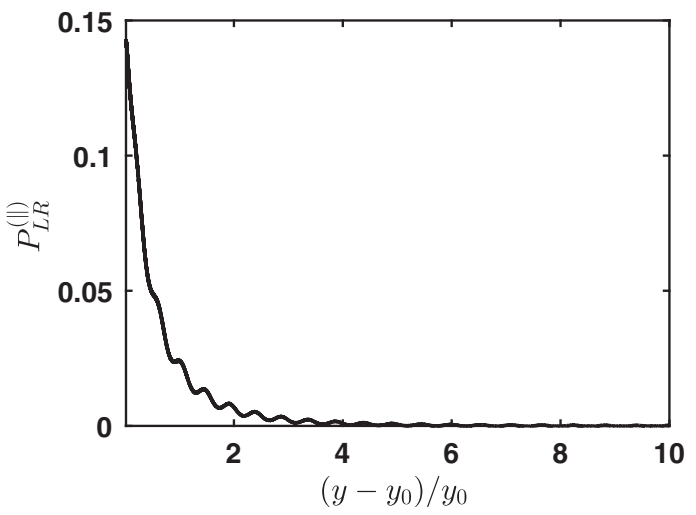

(c)
We assume that the neutrino beam scatters off a SMBH surrounded by an accretion disk. For example, we can suppose that such a SMBH is in the center of a Seyfert galaxy. We take that the plasma density in the disk scales as $n_{e} \propto r^{-\beta}$. The value of $\beta$ is very model dependent. For example, $\beta \approx 0.5$ in an advection dominated accretion disk studied in Ref. [22]. If we take that the mass of the SMBH in question is $M \sim 10^{8} M_{\odot}$, the plasma density in the vicinity of the SMBH can be up to $n_{e} \sim 10^{18} \mathrm{~cm}^{-3}$ [23]. Thus, the dimensionless effective potential $V(r)=G_{\mathrm{F}} n_{e}(r) r_{g} / \sqrt{2}$, reads $V(x)=V_{\max } x^{-\beta}$, where $x=r / r_{g}$.

One can consider various neutrino trajectories with respect to an accretion disk. However, to highlight the effect of the neutrino interaction with matter, we study two extreme cases: the neutrino motion in the plane perpendicular to an accretion disk, marked by the symbol $\perp$, and the neutrino propagation in the plane of an accretion disk, labeled by the symbol $\|$. The effect of the neutrino matter interaction is maximal in the latter situation, since we assume that a disk is slim.

First, we discuss the case $\perp$, when only the gravity contributes to the neutrino scattering off the $\mathrm{BH}$. The transition and survival probabilities, as the functions of

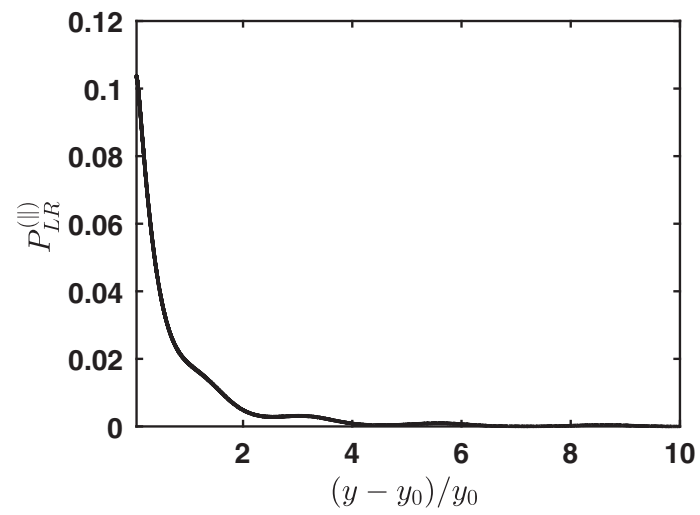

(b)

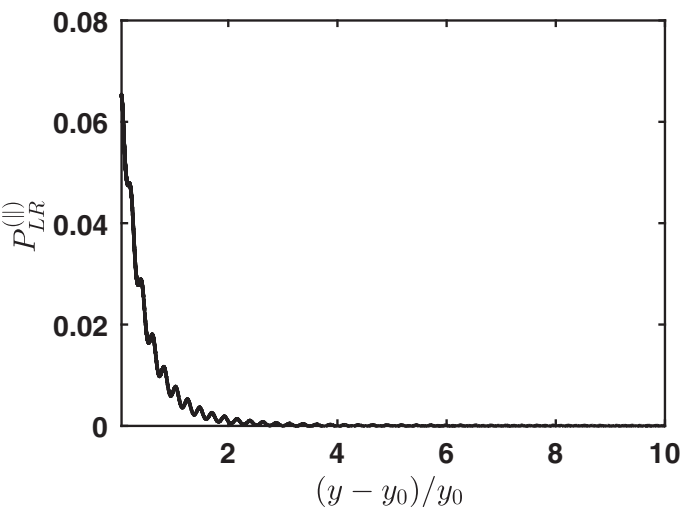

(d)

FIG. 2. The transition probability of spin oscillations $P_{\mathrm{LR}}^{(\|)}$when a neutrino interacts with an accretion disk versus the dimensionless impact parameter $y$. (a) $V_{\max }=0.1\left(n_{e}=10^{18} \mathrm{~cm}^{-3}\right)$ and $\beta=0.5$, (b) $V_{\max }=0.2\left(n_{e}=2 \times 10^{18} \mathrm{~cm}^{-3}\right)$ and $\beta=0.5$, (c) $V_{\max }=0.1$ and $\beta=0.2$, (d) $V_{\max }=0.2$ and $\beta=0.2$. 


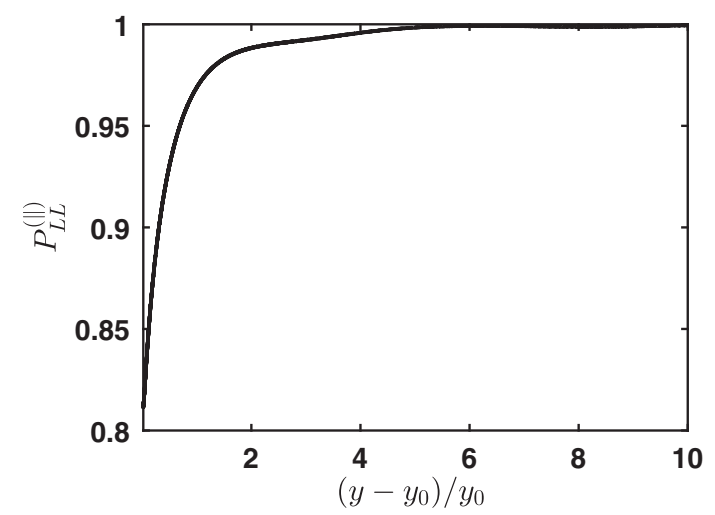

(a)

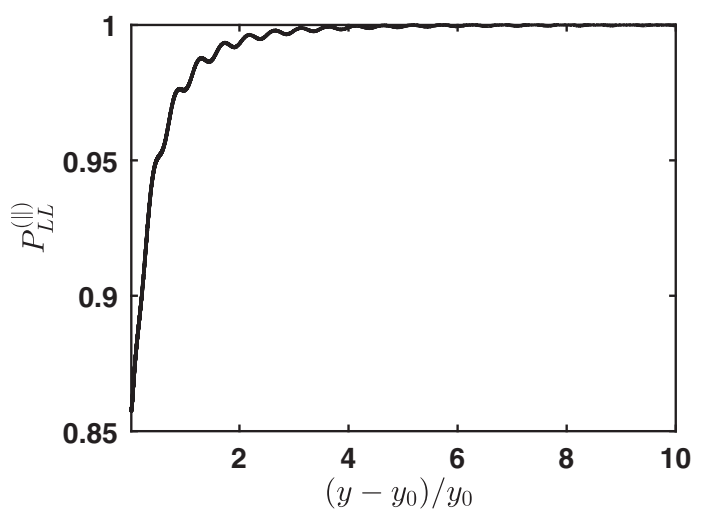

(c)

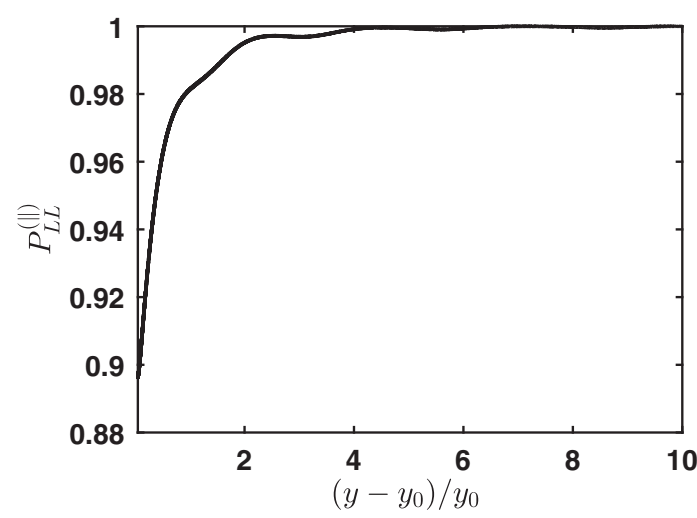

(b)

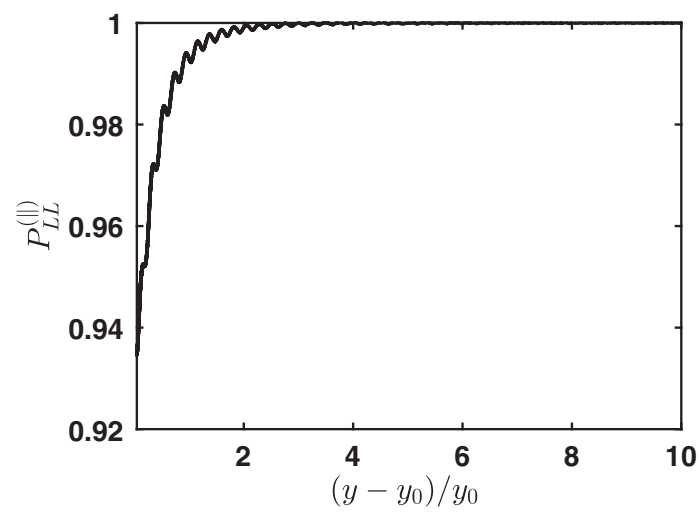

(d)

FIG. 3. The survival probability of spin oscillations $P_{\mathrm{LL}}^{(\|)}$, when a neutrino interacts with an accretion disk, versus the dimensionless impact parameter $y$ for different $V_{\max }$ and $\beta$. (a) $V_{\max }=0.1\left(n_{e}=10^{18} \mathrm{~cm}^{-3}\right)$ and $\beta=0.5$, (b) $V_{\max }=0.2\left(n_{e}=2 \times 10^{18} \mathrm{~cm}^{-3}\right)$ and $\beta=0.5$, (c) $V_{\max }=0.1$ and $\beta=0.2$, (d) $V_{\max }=0.2$ and $\beta=0.2$.

the dimensionless impact parameter $y=b / r_{g}$, are shown in Figs. 1(a) and 1(b). Despite the fact that we show the probabilities for $y_{0}<y<11 y_{0}$ (see also Figs. 2 and 3), we take that $y<30 y_{0}$ in our simulations. One can see in Figs. 1(a) and 1(b) that $P_{\mathrm{LR}}^{(\perp)} \rightarrow 0.25\left(P_{\mathrm{LL}}^{(\perp)} \rightarrow 0.75\right)$ at $y \rightarrow$ $y_{0}$ and $P_{\mathrm{LR}}^{(\perp)} \rightarrow 0\left(P_{\mathrm{LL}}^{(\perp)} \rightarrow 1\right)$ at $y \gg y_{0}$, which is in agreement with the results in Sec. II.

The probabilities as functions of the impact parameter, shown in Figs. 1(a) and 1(b), are not the measurable quantities. In Fig. 1(c), we show the measured flux of neutrinos, moving perpendicular to an accretion disk $F_{\nu}^{(\perp)}$, normalized by the flux of scalar particles versus the scattering angle $\chi$. These fluxes are proportional to the differential cross section, $F \sim \mathrm{d} \sigma / \mathrm{d} \Omega$, where $\mathrm{d} \Omega=2 \pi \sin \chi \mathrm{d} \chi$. The flux of scalar particles (or the cross section) $F_{0}$ is given in Appendix (see also Ref. [24]).

One can see in Fig. 1(c) that spin effects in the neutrino gravitational scattering off the $\mathrm{BH}$ significantly reduce the observed flux of neutrinos compared to the case of scalar particles. The influence of spin oscillations is maximal for neutrinos scattered backward. The reduction of the flux can be more than $20 \%$ in this situation.
Now we turn to the discussion of the neutrino interaction with both the gravity and an accretion disk; i.e., we discuss the case $\|$. The transition and survival probabilities are shown in Figs. 2 and 3 for various $V_{\max }$, or the maximal plasma density $n_{e}$, and $\beta$.

One can see that the best coincidence between $\perp$ and $\|$ cases is implemented when $V_{\max }=0.1$ and $\beta=0.5$ [cf. Figs. 1(a) and 2(a), as well as Figs. 1(b) and 3(a)]. Indeed, this situation corresponds to a low density accretion disk with $n_{e}=10^{18} \mathrm{~cm}^{-3}$, which has the relatively rapid density decrease (great $\beta=0.5$ ); i.e., the influence of the neutrino matter interaction is minimal. The opposite case is presented in Figs. 2(d) and 3(d), where the influence of matter on neutrino spin oscillations is maximal since matter density is higher, $n_{e}=2 \times 10^{18} \mathrm{~cm}^{-3}$. Moreover, the density profile is less steep (small $\beta=0.2$ ); i.e., a neutrino stays longer inside such a disk.

We also mention that the analysis of neutrino spin oscillations in accretion disks with a constant density, studied in Ref. [23], is problematic because of difficulties in the numerical solution of Eqs. (3.3) and (3.4) in the limit $\beta \rightarrow 0$. 


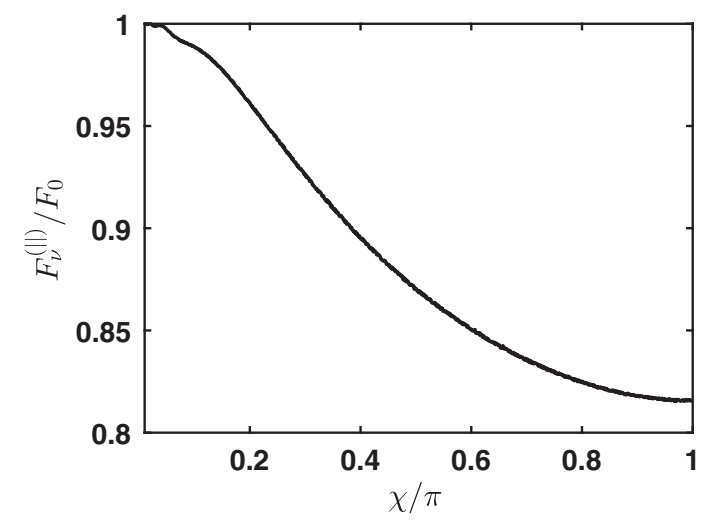

(a)

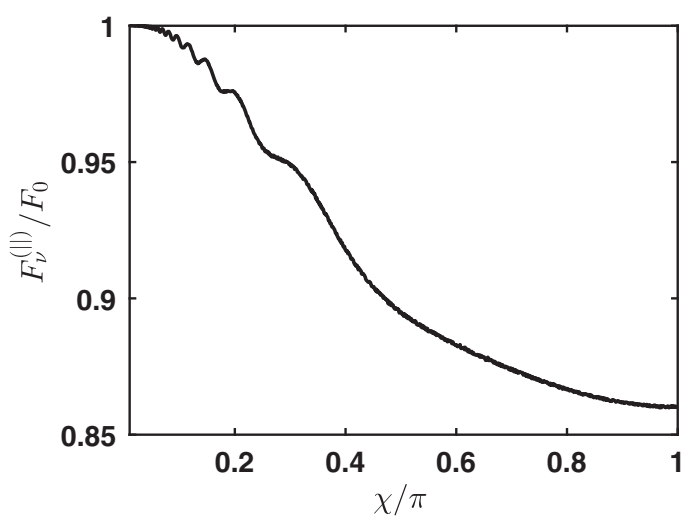

(c)

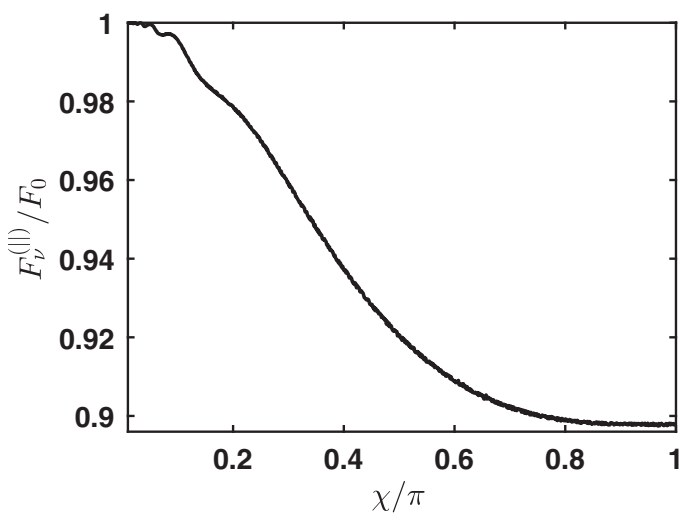

(b)

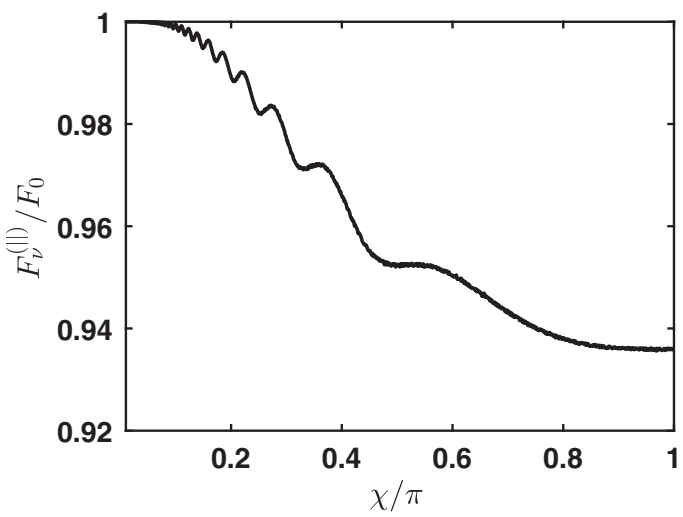

(d)

FIG. 4. The fluxes of neutrinos, normalized by the flux of scalar particles, for neutrinos interacting with background matter of an accretion disk. Here we represent the dependence of $F_{\nu}^{(\|)}$on $\chi$ for different $V_{\max }$ and $\beta$. (a) $V_{\max }=0.1\left(n_{e}=10^{18} \mathrm{~cm}^{-3}\right)$ and $\beta=0.5$, (b) $V_{\max }=0.2\left(n_{e}=2 \times 10^{18} \mathrm{~cm}^{-3}\right)$ and $\beta=0.5$, (c) $V_{\max }=0.1$ and $\beta=0.2$, (d) $V_{\max }=0.2$ and $\beta=0.2$.

We have already mentioned above that the probabilities of spin oscillations versus the impact parameter cannot be measured in an experiment. In Fig. 4, we show the fluxes of neutrinos $F_{\nu}^{(\|)}$scattered off $\mathrm{BH}$ and interacting with an accretion disk. These fluxes are normalized to the flux of scalar particles. As mentioned above, the best coincidence between $\perp$ and $\|$ cases is implemented for $V_{\text {max }}=0.1$ and $\beta=0.5$; cf. Figs. 4(a) and 1(c).

Now, we explicitly compare $\perp$ and $\|$ cases by plotting the ratios of the corresponding fluxes in Fig. 5. First, we mention that $F_{\nu}^{(\perp)}<F_{\nu}^{(\|)}$. Indeed, if a neutrino interacts only with gravity, spin oscillations are in the resonance (see Refs. $[5,6])$. The interaction with matter makes the survival probability greater. This fact explains the observed feature.

The difference between $F_{\nu}^{(\perp)}$ and $F_{\nu}^{(\|)}$can reach almost 20\%; see Fig. 5(d). It means that, if high energy astrophysical neutrinos experience gravitational lensing by a BH surrounded by an accretion disk, the observed flux depends on the orientation of the neutrino trajectory with respect to the disk plane. This maximal difference between $F_{\nu}^{(\perp)}$ and $F_{\nu}^{(\|)}$is for backwardly scattered neutrinos.

\section{DISCUSSION}

In the present work, we have considered spin effects in the neutrino scattering off a nonrotating $\mathrm{BH}$. The neutrino spin evolution in curved spacetime has been accounted for quasiclassically based on the approach developed in Refs. [5,6]. We have studied the neutrino scattering off SMBH surrounded by an accretion disk and considered some astrophysical applications.

In Sec. II, we have studied the neutrino spin evolution in a gravitational scattering in the Schwarzschild metric. Supposing that all incoming neutrinos are ultrarelativistic and left polarized, we have obtained that the transition probability $P_{\mathrm{LR}}$ for outgoing particles can reach $25 \%$ if the impact parameter is close to the critical one $b \approx b_{0}=3 \sqrt{3} r_{g} / 2$. Note that the fact that the helicity of ultrarelativistic, or even massless, fermions can be changed under the influence of a gravitational field was noticed earlier in Ref. [25]. We also mention that our calculation of the probabilities in the limit $y \gg y_{0}$ in Eq. (2.12) is consistent with the result of Ref. [26], where the neutrino helicity flip in the idealized gravitational field was studied. 


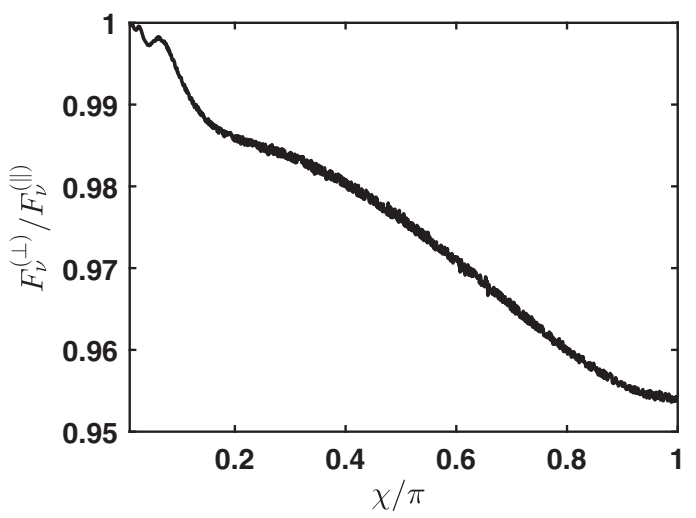

(a)

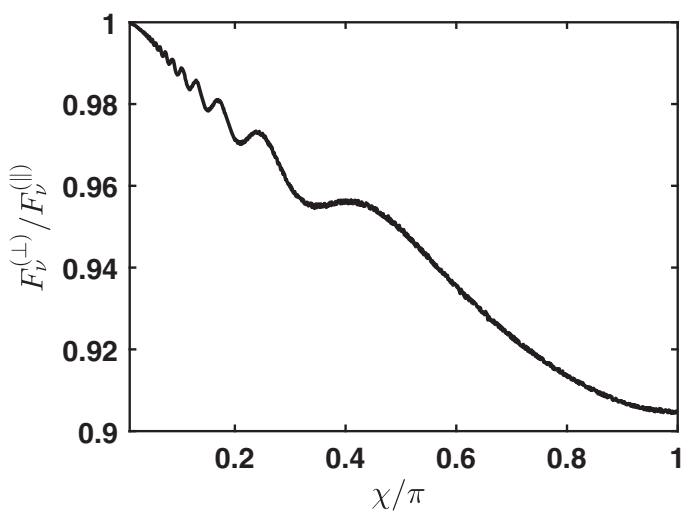

(c)

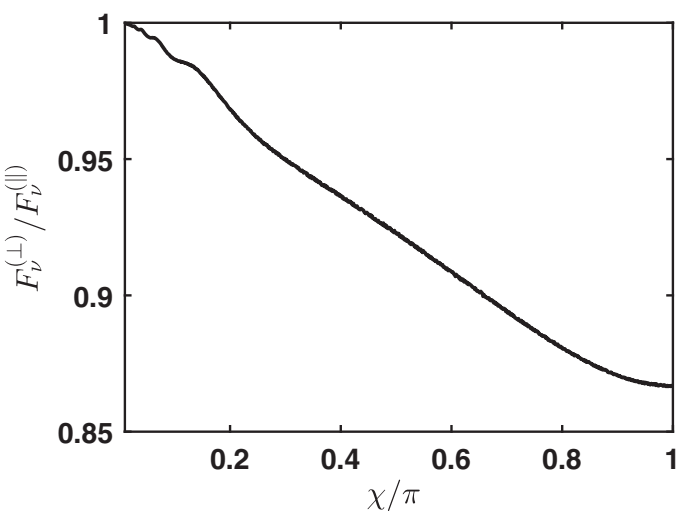

(b)

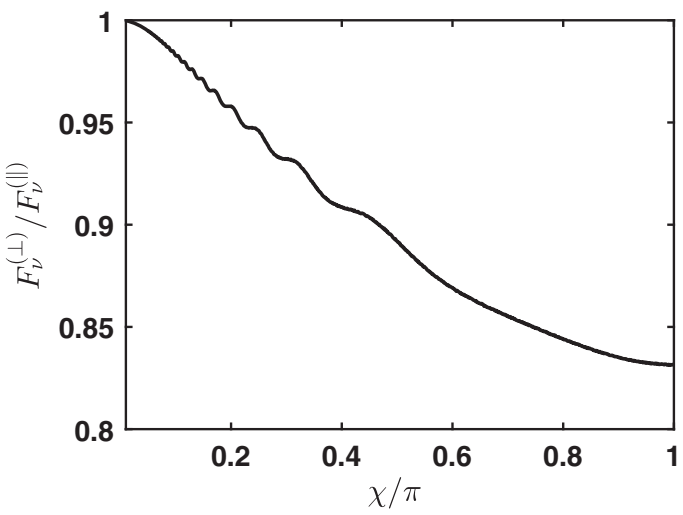

(d)

FIG. 5. The ratio $F_{\nu}^{(\perp)} / F_{\nu}^{(\|)}$versus the scattering angle $\chi$ for different $V_{\max }$ and $\beta$. (a) $V_{\max }=0.1\left(n_{e}=10^{18} \mathrm{~cm}^{-3}\right)$ and $\beta=0.5$, (b) $V_{\max }=0.2\left(n_{e}=2 \times 10^{18} \mathrm{~cm}^{-3}\right)$ and $\beta=0.5$, (c) $V_{\max }=0.1$ and $\beta=0.2$, (d) $V_{\max }=0.2$ and $\beta=0.2$.

Then, in Sec. III, we have derived the effective Schrödinger equation for a neutrino scattering off a $\mathrm{BH}$ surrounded by background matter with a nonuniform density. In the case of only gravitational scattering, studied in Sec. II, it was possible to obtain the analytical transition and survival probabilities for some impact parameters. If, in addition to gravity, a neutrino interacts with a background matter, the probabilities can be derived only in the numerical solution of Eqs. (3.3) and (3.4).

In Sec. IV, we have considered the astrophysical applications of our results. In particular, we have studied the effect of spin oscillations on the neutrino scattering off SMBH surrounded by the accretion disk. We have taken the parameters of the accretion disk, such as the maximal number density and the profile of the mass distribution, close to the values resulting from observations and hydrodynamics simulations. Using the numerical solution of Eqs. (3.3) and (3.4), we have found the transition and survival probabilities, as well as the observed fluxes of outgoing neutrinos for different orientations of the particles trajectories with respect to the accretion disk.

As one can see in Figs. 1(c) and 4, there is no deviation of the fluxes for the forward neutrino scattering at $\chi=0$ if one compares them with the fluxes of scalar particles. It means that neutrino spin oscillations do not affect the size of the BH shadow. The major effect of spin oscillations is for the backward neutrino scattering at $\chi=\pi$. Thus, the intensity of the glory flux for neutrinos is almost $20 \%$ less than for scalar particles; cf. Fig. 4(a).

The influence of the plasma interaction on the gravitational scattering of scalar particles (photons) was extensively studied (see, e.g., Ref. [18] for a review). For example, the photons propagation in plasma surrounding a nonrotating $\mathrm{BH}$ was examined in Ref. [27]. The form of the $\mathrm{BH}$ shadow was found to be unchanged, but its size can be magnified. In Fig. 5, we predict the asymmetry in the observed neutrino fluxes depending on the orientation of the neutrino trajectory with respect to the accretion disk. This asymmetry is maximal for the backward neutrino scattering. Although neutrinos interact with plasma much weaker than photons, the asymmetry can reach almost $20 \%$ for the realistic accretion disk; cf. Fig. 5(d).

Since the effects of spin oscillations on the neutrino gravitational scattering are valid for ultrarelativistic particles, the results obtained in the present work are of importance for the rapidly developing area of neutrino astronomy [28], where significant success was achieved in 
the detection of the ultrahigh energy (UHE) cosmic neutrinos. Neutrinos with energies in the $\mathrm{PeV}$ range were reported in Ref. [29] to be recorded. Moreover, there are sizable efforts in the identification of the sources of UHE neutrinos with astronomical objects, such as active galactic nuclei [30]. In our work, we have demonstrated that, if the incoming flux of cosmic neutrinos experience the gravitational lensing, the observed flux can be reduced by down to $20 \%$ compared to its initial value because of neutrino spin oscillations.

\section{ACKNOWLEDGMENTS}

I am thankful to J. Jiang, Y. N. Obukhov, and A. F. Zakharov for useful comments. This work is performed within the government assignment of IZMIRAN. I am also thankful to RFBR (Grant No. 18-02-00149a) and DAAD for partial support.

\section{APPENDIX: PARTICLE MOTION IN THE SCHWARZSCHILD METRIC}

In this Appendix, we briefly remind the reader how to describe the motion of a scalar particle interacting with a nonrotating $\mathrm{BH}$, as well as how to calculate the differential cross section of the gravitational scattering. These problems were studied in detail in Refs. ([19], pp. 287-290, and [24]).

The energy $E$ and the angular momentum $L$ are the conserved quantities for a particle with the mass $m$ moving in the Schwarzschild metric in Eq. (2.1). The equation of motion and the trajectory are defined by

$$
\begin{aligned}
\frac{\mathrm{d} r}{\mathrm{~d} t} & = \pm \frac{m A^{2}}{E}\left[\frac{E^{2}}{m^{2}}-A^{2}\left(1+\frac{L^{2}}{m^{2} r^{2}}\right)\right]^{1 / 2}, \\
\frac{\mathrm{d} \phi}{\mathrm{d} r} & = \pm \frac{L}{m r^{2}}\left[\frac{E^{2}}{m^{2}}-A^{2}\left(1+\frac{L^{2}}{m^{2} r^{2}}\right)\right]^{-1 / 2},
\end{aligned}
$$

for a particle moving in the equatorial plane. In Eq. (A1), the minus signs stay for incoming particles and the plus signs stay for outgoing particles.

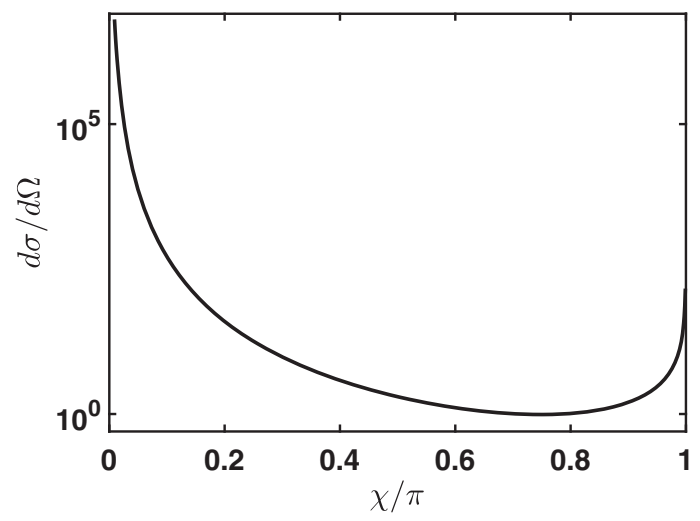

FIG. 6. The differential cross section of the gravitational scattering of scalar particles off a nonrotating $\mathrm{BH}$, normalized by $r_{g}^{2}$, versus the angle $\chi$.

If we study ultrarelativistic particles, the angle corresponding to the minimal distance between a particle and $\mathrm{BH}$ is

$$
\phi_{m}=y \int_{x_{m}}^{\infty} \frac{\mathrm{d} x}{\sqrt{x\left[x^{3}-y^{2}(x-1)\right]}},
$$

where $y=b / r_{g}, \quad b=L / E$ is the impact parameter, and $x_{m}$ is the maximal root of Eq. (2.9). The parameter $y>y_{0}=3 \sqrt{3} / 2$. Otherwise a particle falls to $\mathrm{BH}$.

While computing the differential cross section, $\mathrm{d} \sigma / \mathrm{d} \Omega$, where $\mathrm{d} \Omega=2 \pi \sin \chi \mathrm{d} \chi$, we should take into account that a particle, before being scattered off, can make multiple revolutions around a $\mathrm{BH}$, in both clockwise and anticlockwise directions. One should account for this fact in the determination of the angle $\chi$, fixing the position of a detector, which is in the range $0<\chi<\pi$.

In Fig. 6, we present the result of the numerical computation of the cross section. While building this plot, we take that $y_{0}<y<30 y_{0}$ and account for up to two revolutions of a particle around the $\mathrm{BH}$ in both directions. This result is used in Sec. IV when we study the neutrino scattering off a realistic $\mathrm{BH}$ surrounded by an accretion disk.
[1] M. Agostini et al. (Borexino Collaboration), Comprehensive measurement of $p p$-chain solar neutrinos, Nature (London) 562, 505 (2018).

[2] C. Giunti and A. Studenikin, Neutrino electromagnetic interactions: A window to new physics, Rev. Mod. Phys. 87, 531 (2015).

[3] M. Maltoni and A. Yu. Smirnov, Solar neutrinos and neutrino physics, Eur. Phys. J. A 52, 87 (2016).
[4] D. V. Ahluwalia and C. Burgard, Gravitationally induced neutrino-oscillation phases, Gen. Relativ. Gravit. 28, 1161 (1996).

[5] M. Dvornikov, Neutrino spin oscillations in gravitational fields, Int. J. Mod. Phys. D 15, 1017 (2006).

[6] M. Dvornikov, Neutrino spin oscillations in matter under the influence of gravitational and electromagnetic fields, J. Cosmol. Astropart. Phys. 06 (2013) 015. 
[7] M. Dvornikov, Neutrino spin oscillations in external fields in curved spacetime, Phys. Rev. D 99, 116021 (2019).

[8] Yu. N. Obukhov, A. J. Silenko, and O. V. Teryaev, Spin dynamics in gravitational fields of rotating bodies and the equivalence principle, Phys. Rev. D 80, 064044 (2009).

[9] Y. N. Obukhov, A. J. Silenko, and O. V. Teryaev, General treatment of quantum and classical spinning particles in external fields, Phys. Rev. D 96, 105005 (2017).

[10] F. Sorge and S. Zilio, Neutrino spin flip around a Schwarzschild black hole, Classical Quantum Gravity 24, 2653 (2007).

[11] S. A. Alavi and S. Nodeh, Neutrino spin oscillations in gravitational fields in noncommutative spaces, Phys. Scr. 90, 035301 (2015).

[12] S. Chakraborty, Aspects of neutrino oscillation in alternative gravity theories, J. Cosmol. Astropart. Phys. 10 (2015) 019.

[13] K. Akiyama et al. (Event Horizon Telescope Collaboration), First M87 event horizon telescope results. I. The shadow of the supermassive black hole, Astrophys. J. Lett. 875, L1 (2019).

[14] S. E. Gralla, D. E. Holz, and R. M. Wald, Black hole shadows, photon rings, and lensing rings, Phys. Rev. D 100, 024018 (2019).

[15] O. L. Caballero, G. C. McLaughlin, and R. Surman, Neutrino spectra from accretion disks: Neutrino general relativistic effects and the consequences for nucleosynthesis, Astrophys. J. 745, 170 (2012).

[16] C. Corianò, A. Costantini, M. Dell'Atti, and L. Delle Rose, Neutrino and photon lensing by black holes: Radiative lens equations and post-Newtonian contributions, J. High Energy Phys. 07 (2015) 160.

[17] Z. Stuchlík and J. Schee, Shadow of the regular Bardeen black holes and comparison of the motion of photons and neutrinos, Eur. Phys. J. C 79, 44 (2019).

[18] P. V. P. Cunha and C. A. R. Herdeiro, Shadows and strong gravitational lensing: A brief review, Gen. Relativ. Gravit. 50, 42 (2018).
[19] L. D. Landau and E. M. Lifschitz, The Classical Theory of Fields, 3rd ed. (Pergamon Press, Oxford, 1971).

[20] R. N. Mohapatra and P. B. Pal, Massive Neutrinos in Physics and Astrophysics, 3rd ed. (World Scientific, Singapore, 2004), p. 98.

[21] M. Dvornikov and A. Studenikin, Neutrino spin evolution in presence of general external fields, J. High Energy Phys. 09 (2002) 016.

[22] I. V. Igumenshchev, M. A. Abramowicz, and R. Narayan, Numerical simulations of convective accretion flows in three dimensions, Astrophys. J. 537, L27 (2000).

[23] J. Jiang, A. C. Fabian, T. Dauser, L. Gallo, J. A. Garcia, E. Kara, M. L. Parker, J. A. Tomsick, D. J. Walton, and C. S. Reynolds, High density reflection spectroscopy-II. The density of the inner black hole accretion disc in AGN, Mon. Not. R. Astron. Soc. 489, 3436 (2019).

[24] S. Dolan, C. Doran, and A. Lasenby, Fermion scattering by a Schwarzschild black hole, Phys. Rev. D 74, 064005 (2006).

[25] D. Singh, N. Mobed, and G. Papini, Helicity precession of spin-1/2 particles in weak inertial and gravitational fields, J. Phys. A 37, 8329 (2004).

[26] C. Mergulhão, Jr., Neutrino helicity flip in a curved spacetime, Gen. Relativ. Gravit. 27, 657 (1995).

[27] V. Perlick and O. Yu. Tsupko, Light propagation in a plasma on Kerr spacetime: Separation of the Hamilton-Jacobi equation and calculation of the shadow, Phys. Rev. D 95, 104003 (2017).

[28] A. G. Rosso, C. Mascaretti, A. Palladino, and F. Vissani, Introduction to neutrino astronomy, Eur. Phys. J. Plus 133, 267 (2018).

[29] M. G. Aartsen et al. (IceCube Collaboration), First Observation of PeV-Energy Neutrinos with IceCube, Phys. Rev. Lett. 111, 021103 (2013).

[30] M. G. Aartsen et al. (IceCube Collaboration), TimeIntegrated Neutrino Source Searches with 10 Years of IceCube Data, Phys. Rev. Lett. 124, 051103 (2020). 